\title{
DNA sequence polymorphism of the $R h g 4$ candidate gene conferring resistance to soybean cyst nematode in Chinese domesticated and wild soybeans
}

\author{
Cui-Ping Yuan • Ying-Hui Li • \\ Zhang-Xiong Liu $\cdot$ Rong-Xia Guan • \\ Ru-Zhen Chang $\cdot$ Li-Juan Qiu
}

Received: 20 August 2011/Accepted: 21 January 2012/Published online: 18 February 2012

(C) The Author(s) 2012. This article is published with open access at Springerlink.com

\begin{abstract}
Rhg4 is one of the major resistant genes conferring resistance to soybean cyst nematode races 1 , 3 and 4 . In order to better understand its sequence diversity among different Chinese soybean populations and the impact of human activities on it, we designed 5 primer sets based on its sequence deposited in Genbank (Genbank accession No. AF506518) to obtain the Rhg4 sequence from 104 Chinese cultivated and wild soybean genotypes, and then analyzed the DNA sequence polymorphism in different Chinese soybean populations. The alignment of Rhg4 sequence included 5,216 nucleotide base pairs. A total of 67 single nucleotide polymorphisms (SNPs) including 59 single base changes and 8 DNA insertion-deletions (InDels) were
\end{abstract}

Electronic supplementary material The online version of this article (doi:10.1007/s11032-012-9703-1) contains supplementary material, which is available to authorized users.

C.-P. Yuan · Y.-H. Li · Z.-X. Liu · R.-X. Guan ·

R.-Z. Chang · L.-J. Qiu ( $₫)$

The National Key Facility for Crop Gene Resources and Genetic Improvement (NFCRI), Institute of Crop

Sciences, Chinese Academy of Agricultural Sciences, Beijing 100081, China

e-mail: qiu_lijuan@263.net; qiu_lijuan@mail.caas.net.cn

C.-P. Yuan

e-mail: cpyuan2004@126.com

C.-P. Yuan

Presently Working at the Biotechnology Research Centre, Jilin Academy of Agricultural Sciences, Changchun 130033, China identified with a SNP frequency of 1/78. Except for a 14-base InDel, there were 29 SNPs in coding regions, and among them, 13 were non-synonymous ( 9 in functional domains with 1 in a leucine-rich repeats region, 2 in a transmembrane region and 6 in a Ser/Thr kinase domain). The probability of substitution at each site was not the same, there were two hot spots, one was in the $5^{\prime}$-untranslated region between positions 124 and 804 , and the other was in the region between positions 2520 and 3733 . Sequence diversity analysis among 104 soybean genotypes showed $\pi=0.00102$ and $\theta=0.00218$ for Rhg4. A domestication bottleneck was found because of lower sequence diversity and 58\% unique SNPs loss in landraces compared with Glycine soja. Intensive selection increased the sequence diversity of cultivars, which had higher diversity and more unique SNPs than landraces.

Keywords Glycine max - Glycine soja - Sequence diversity $\cdot$ Heterodera glycines

\section{Introduction}

Soybean cyst nematode (SCN) (Heterodera glycines Ichinohe) is one of the most devastating pathogens in soybean production worldwide and causes substantial yield losses ( $\mathrm{Li}$ et al. 2011; Wrather et al. 2001; Wrather and Koenning 2009) by feeding on soybean roots, damaging root systems, and reducing the plant's ability to absorb water and nutrients. Resistant 
cultivars are considered the best method to control SCN. Many scientists have conducted research programs in an effort to identify resistant sources (Arelli and Wilcox 1997; Arelli et al. 2000; Coordinative group of evaluation of SCN 1993; Lai et al. 2005; Young 1990; Zhang and Dai 1992), identify the genes involved (Caldwell et al. 1960; Lu et al. 2006; Matson and Williams 1965; Rao-Arelli et al. 1992; Vuong et al. 2010; Wang et al. 2001; Winter et al. 2007) or produce resistant varieties (Anand et al. 2004; Diers et al. 2006; Du et al. 2006; Hao et al. 2003; Mengistu et al. 2005; Qiu and Wang 2007; Shannon et al. 2009; Wang et al. 2007). Classical inheritance studies identified five SCN resistance genes in soybean, three recessive genes designated $r h g 1, r h g 2$ and $r h g 3$ were first reported in 'Peking' (Caldwell et al. 1960), the dominant gene Rhg4 was also identified in 'Peking', and was linked to the ' $i$ ' locus controlling seed coat color (Matson and Williams 1965), an additional dominant gene, $R h g 5$, was reported in PI 88788 (RaoArelli et al. 1992).

Almost 20 years of genetic mapping studies described more than $70 \mathrm{SCN}$ resistance quantitative trait loci (QTLs) (Concibido et al. 2004; Guo et al. 2006; Vuong et al. 2010; Winter et al. 2007; Wu et al. 2009; Yuan et al. 2006). Despite inconsistencies of QTL mapping, it was concluded that $r h g 1$ and $R h g 4$ were two major SCN resistance genes. The rhgl locus repeatedly mapped on linkage group (LG) G [chromosome (Chr) 18] in many resistant soybean genotypes (Chang et al. 1997; Concibido et al. 1994, 1997; Guo et al. 2006; Prabhu et al. 1999; Webb et al. 1995; Yue et al. 2001) and provided the greatest level of resistance. Ruben et al. (2006) summarized the construction of integrated physical and genetic maps of a $0.2 \mathrm{cM}$ interval encompassing the rhgl locus, and characterized the candidate gene as well as the encoding protein, RHG1, a receptor-like kinase. Li et al.(2009) developed 6 SNP markers based on the variation in rhgl and reported their significant improvement of efficiency in marker-assisted selection (MAS) when combined with microsatellite marker BACR-Satt309, although Melito et al. (2010) reported no significant impacts of the LRRkinase gene on SCN resistance.

Meanwhile Rhg4 was located on LG A2 (Chr 8) (Chang et al. 1997; Concibido et al. 1994; Guo et al. 2006; Heer et al. 1998; Mahalingam and Skorupska 1995; Webb et al. 1995), $0.35 \mathrm{cM}$ from the $I$ locus (Matson and Williams 1965). Several genes associated with stress or defense responses such as chalcone synthase, glucosyl-transferase, heat-shock transcription factor, protein kinase, G10-like protein and restriction fragment length polymorphism molecular marker pBLT65 were close to the I and Rhg 4 loci (Heer et al. 1998; Lewers et al. 2002; Matthews et al. 2001; Todd and Vodkin 1996; Webb et al. 1995; Weismann et al. 1992). Two separate research groups isolated the receptor-like kinase candidate gene Rhg4 from soybean variety 'Forrest' by positional cloning (Hauge et al. 2001; Lightfoot and Meksem 2002) and its DNA and protein sequence were lodged in Genbank in 2002 (Genbank accessions AF506518 and AAM44275.1). However, the candidate gene was little studied except for the work of Jang et al. (2004) who reported 3 SNPs and 7 InDels within two regions of Rhg 4 totalling 901 bp by direct sequencing with 2 primer sets.

Like other important crops, soybean has undergone selection by human, involving domestication, intensive breeding, and probable founding events (Gyuhwa and Ram 2008; Hyten et al. 2006). These selection activities likely decrease genetic diversity (Tenaillon et al. 2001; Zhu et al. 2007), change allelic frequencies (Hyten et al. 2006) and eliminate rare alleles (Hyten et al. 2006; Tenaillon et al. 2001). Cultivated soybean (G. $\max$ ) was domesticated from wild soybean (G. soja) in China (Hymowitz and Newell 1981), and domestication immediately resulted in G. max landraces (Hyten et al. 2007). Subsequent intensive selection imposed on landraces by soybean breeding created elite soybean cultivars. Hyten et al. (2006) and Yuan et al. (2008) detected effects of domestication bottlenecks in soybeans, but they had inconsistent results regarding intensive selection effects. Hyten et al. (2006) showed that modern soybean breeding had only minimal affects on the allelic structure of the soybean genome, but Yuan et al. (2008) reported an intensive selection bottleneck on $\mathrm{GmHs} \mathrm{I}^{\text {pro-1}}$. However, in investigating the founding effects of soybean introduction to North America, they found evidence for only minor and non- significant bottlenecks (Hyten et al. 2006). The cumulative effects of the two genetic bottlenecks caused by founding events and intensive selection led to significant reductions in genetic diversity among North American elite cultivars in comparison with Asian landraces (Hyten et al. 2006).

In the present work we quantified DNA sequence polymorphism of Rhg4 in Chinese domesticated and wild soybeans by investigating an almost complete 
Rhg4 gene sequence in order to better understand its sequence diversity among different Chinese soybean populations and the impact of human activities on the candidate resistance gene. The resulting information may help the development of SNP markers for use in MAS in breeding programs.

\section{Materials and methods}

\section{Plant materials}

The plant materials were selected from 27 provinces (autonomous regions or municipalities) of China (MOESM1) and represent three populations ( $G$. soja, landraces and cultivars). The population of $G$. soja consisted of 28 accessions from 14 provinces (autonomous regions), landraces were represented by 51 accessions from 22 provinces (autonomous regions or municipalities), cultivars were from 7 provinces (municipalities), including 8 soybean genotypes from our core collection resistant to SCN (Ma et al. 2006), 3 awarded varieties, 4 parental lines (varieties) of our soybean genetic populations, and 10 elite cultivars. Genomic DNA was extracted from seedlings of wild accessions and from seed of domesticated genotypes as described by Yuan et al. (2008).

Primers design and polymerase chain reaction (PCR) amplification

Five pairs of primers (MOESM2) were designed from the sequence of the Glycine $\max$ receptor-like kinase Rhg4 gene (GenBank accession AF506518), with overlaps of $137-218$ bp between adjacent PCR regions. PCR was carried out in total volumes of $20 \mu \mathrm{l}$ consisting of $60 \mathrm{ng}$ DNA, $1 \times$ PCR TaKaRa Buffer, $0.15 \mu \mathrm{M}$ of each primer, $0.15 \mathrm{mM}$ dNTP and $0.5 \mathrm{U}$ TaKaRa ExTaq polymerase. PCR amplification conditions were as described by Yuan et al. (2008).

\section{Sequencing}

PCR products were separated on $1.0 \%$ agarose gels stained with ethidium bromide. The PCR primer set of $3561 \mathrm{U} 22$ and 4782L26 produced 3 amplicons with about 90 nucleotide base differences in length. A preexperiment determined that the smallest amplicon was the most similar to AF506518 and located in LG A2 (Chr 8) by blastn against AF506518 (unshown), so we chose the smallest amplicon as a target fragment. The fragment was purified using a DNA fragment purification kit (Biotech), cloned into the pMD18-T vector (TaKaRa) and then sequenced with primers of M13F and M13R. The other 4 PCR primer sets each produced single amplicons, and the PCR products were directly sequenced with PCR primers after being collected and purified. When necessary, sequencing primers (MOESM2) were designed from the sequencing information to assure accuracy of sequence determination.

Sequence analysis

Sequences were assembled with the SeqMan tool of DNAstar software, and the sequence of cv. Huipizhiheidou (HPZhHD) was interrogated by BLAST searches in Genbank for identity confirmation. Sequence alignment was performed using Clustalx 1.8 with manual refinement. Single base changes and single or multiple base InDels were collectively preferred as SNPs. Only informative SNP sites were selected to build haplotypes. Differential regions of the DNA sequence were predicted and located following the method of Tang and Lewontin (1999). Two DNA polymorphism measures of nucleotide diversity ( $\pi$ and $\theta$ ) and haplotype diversity were calculated with DnaSP v.5.10.01 software. A neighbor-joining phyolgenetic tree was constructed using MEGA 5.0 software with a Kimura 2-parameter model and 1,000 bootstrap replications.

\section{Results}

Sequence comparison of Rhg4 with AF506518

Sequence assembly with the SeqMan program generated a continous sequence of 5,216 bp and a BLAST search showed it had $98 \%$ identity at the nucleotide level with the receptor-like kinase Rhg4 gene (AF506518), therefore the sequence was presumed to be Rhg4. Alignment with AF506518, there were 49 base changes, 2 single-base inserts and one three-base insert (MOESM3). Both single-base inserts occurred in the $5^{\prime}$-untranslated region (UTR), whereas the three- 
base insert was in the second exon, but it was not a frame-shift mutation. The predicated protein therefore had one more amino acid than the receptor-like kinase RHG4 (AAM44275.1).

\section{DNA variants of $R h g 4$}

We obtained the 5,216 bp DNA sequence of $R h g 4$ from 104 soybean genotypes, representing members of the three distinct populations, viz. G. soja, landraces and cultivars. Surprisingly high sequence polymorphism was found in Rhg4, a total of 67 SNPs, including 59 single base changes and 8 DNA InDels, were identified with an SNP frequency of $1 / 78$ (Table 1). Five InDel loci had 3 alleles each, and a 14-base gap occurred in the coding region of one wild soybean genotype presumably leading to a frame-shift mutation. Except for the 14-base Indel, there were 29 SNPs in coding regions, among them 13 were nonsynonymous and 16 were synonymous. Of the 59 single base changes, 40 were involved in transitions, and 19 were transversions with a transition:transversion ratio of $2: 1$.

The predicted RHG4 protein consisted of 894 amino acids, characterized with 3 functional domains of 6 extracellular leucine-rich repeats (LRRs) (56-401), a transmembrane (TM) domain (449-471) and an intracellular Ser/Thr kinase (STK) domain (544-820). Of the 13 non-synonymous nucleotide substitutions, 9 occurred in functional domains, with 1 in the LRR region, 2 in the TM region and 6 in the STK domain.

The probability of substitution at each site in $R h g 4$ was not the same, there were two hot spots with higher probabilities of substitution. One hot spot occurred in the 5'-UTR region between positions 124 and 804 and the other was in the region between positions 2,520 and 3,733 .
DNA polymorphism of Rhg4 among the three soybean populations

Unique and shared SNPs among the three soybean populations were investigated (Fig. 1). A total of 41 SNPs were detected in G. soja, of which 26 were unique and not found in the two G. $\max$ populations. Landraces contained 27 SNPs, of which 11 were unique. Cultivars also had 26 SNPs, 13 of which were unique. While examining the coding region of Rhg4, we found that cultivars had the largest number of sequence variants with 19 SNPs, 11 of which were unique; however G. soja and landraces had 13 and 12 SNPs, with only 7 and 4 , respectively being unique.

Nucleotide diversity analysis on 104 soybean genotypes showed $\pi=0.00102$ and $\theta=0.00218$ for Rhg4 (Table 2). Among the three populations, G. soja had the highest diversity with $\pi=0.00114$ and $\theta=0.00164$, followed by cultivars, and landraces had the lowest with $\pi=0.00090$ and $\theta=0.00098$. Sequence diversity in different regions of the gene showed obvious differences, the highest sequence diversity occurred in synonymous sites of the coding region, followed by intron, the $5^{\prime}$-UTR region, and in non-synonymous sites of the coding region, with the

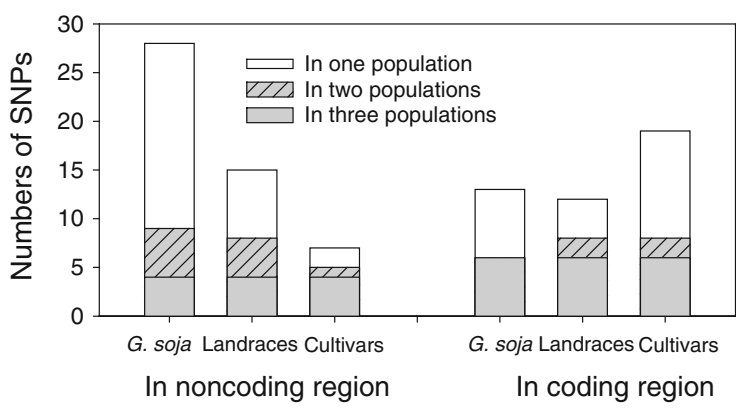

Fig. 1 Number of shared and unique SNPs in the three soybean populations
Table 1 Numbers of SNPs in Rhg4 among 104 soybean genotypes

\begin{tabular}{lccccc}
\hline & $5^{\prime}$-UTR & Coding region & Intron & $3^{\prime}$-UTR & Total \\
\hline InDels & 6 & 1 & 1 & 0 & 8 \\
Single base changes & & & & & \\
$\quad$ Transitions & 6 & 22 & 12 & 0 & 40 \\
$\quad$ Transversions & 6 & 29 & 4 & 2 & 19 \\
$\quad$ Total & 12 & 30 & 17 & 2 & 59 \\
Total & 18 & & & 2 & 67 \\
\hline
\end{tabular}


Table 2 Nucleotide diversity for $R h g 4\left(\times 10^{3}\right)$ in the soybean populations

\begin{tabular}{|c|c|c|c|c|c|c|c|c|c|c|c|c|c|c|}
\hline \multirow[t]{2}{*}{ Population } & \multicolumn{2}{|c|}{$5^{\prime}$-UTR } & \multicolumn{6}{|c|}{ Coding region } & \multicolumn{2}{|c|}{ Intron } & \multicolumn{2}{|c|}{$3^{\prime}$-UTR } & \multicolumn{2}{|c|}{ Total } \\
\hline & $\pi$ & $\theta$ & $\pi($ sny $)$ & $\theta$ (sny) & $\pi$ (Nsny) & $\theta$ (Nsny) & $\pi($ total $)$ & $\theta($ total $)$ & $\pi$ & $\theta$ & $\pi$ & $\theta$ & $\pi$ & $\theta$ \\
\hline \multicolumn{15}{|l|}{ G. $\max$} \\
\hline Landraces & 0.91 & 0.96 & 2.49 & 2.41 & 0.69 & 0.55 & 1.12 & 0.99 & 0.89 & 1.87 & 0.00 & 0.00 & 0.90 & 0.98 \\
\hline Cultivars & 0.86 & 0.46 & 3.33 & 4.11 & 0.89 & 1.17 & 1.48 & 1.88 & 0.86 & 1.11 & 0.00 & 0.00 & 1.07 & 1.22 \\
\hline Total & 0.88 & 0.88 & 2.74 & 3.80 & 0.75 & 1.10 & 1.22 & 1.75 & 0.87 & 2.29 & 0.00 & 0.00 & 0.95 & 1.41 \\
\hline G. soja & 1.31 & 2.24 & 2.82 & 2.81 & 0.71 & 0.63 & 1.21 & 1.16 & 1.47 & 3.24 & 0.22 & 0.78 & 1.14 & 1.64 \\
\hline Total & 1.05 & 2.01 & 2.79 & 4.79 & 0.75 & 1.23 & 1.24 & 2.08 & 1.04 & 4.30 & 0.06 & 0.58 & 1.02 & 2.18 \\
\hline
\end{tabular}

lowest sequence diversity in the $3^{\prime}$-UTR. Cultivars had the highest sequence diversity in the coding region among the three populations.

Three haplotypes of Rhg4 commonly occurred among the three soybean populations, the other 23 haplotypes were in only one or two of the populations. Among the 23 haplotypes, 14 were uniquely detected in $G$. soja, 5 were uniquely in cultivars, 3 were uniquely in landraces, and 1 was detected in both cultivars and landraces. As for haplotype diversity, G. soja had the highest haplotype diversity at 0.97 , whereas cultivars and landraces had lower values (0.89 and 0.80 , respectively).

\section{Discussion}

The SNP frequency in Rhg4 among the 104 soybean accessions was $1 / 78$, obviously higher than earlier estimates of 1/106 (Yuan et al. 2008), 1/107 (Hyten et al. 2006), 1/273 (Zhu et al. 2003) and 1/343 (Van et al. 2005) in soybean. When compared with other plants, the SNP frequency was lower than those reported in maize (Ching et al. 2002; Tenaillon et al. 2001; Yamasaki et al. 2005) and chickpea (Rajesh and Muehlbauer 2008), and higher than those in bread wheat (Ravel et al. 2007) and Arabidopsis thaliana (Schmid et al. 2003; Clark et al. 2007). The difference for SNP frequencies maybe due to different samples and different genomic regions. Although higher SNP frequency was detected than other reports for soybean, sequence diversity among the 3 soybean populations was obviously low compared with other reports among corresponding soybean populations (Hyten et al. 2006). The reason probably was that soybean resources we used were only from China, having somewhat narrower genetic variation.
As for nucleotide mutation type, there is a clear transition bias probable due of the high spontaneous rate of deamination of 5-methl cytosine to thymidine in the CpG dinucleotides (Vignal et al. 2002), that is, GC content is probably linked to the ratio of transitions to transversions. In our study the ratio was 2.1, similar to 2.12 reported in soybean by Van et al. (2005), 2 reported in humans (Wang et al. 1998) and mouse (Lindblad-Toh et al. 2000), and relatively greater than 1.3 reported in soybean (Yuan et al. 2008), but was contrary to 0.93 reported by Zhu et al. (2003) in soybean. The difference among different species and even among different samples in the same species was probably related with GC content in observed genomic region. Of course some other factors leading to DNA mutation may influence the ratio.

Domestication represents the first result of human selection in soybean. Hyten et al. (2006) reported that landraces retained only $66 \%(\pi)$ and $49 \%(\theta)$ of the nucleotide diversity found in G. soja, and had lost $81 \%$ of the rare alleles in $G$. soja, thus representing a domestication bottleneck. Considering the overall sequence region of $R h g 4$, we also found a domestication bottleneck because firstly the sequence diversity within landraces was obviously low $(\pi=0.00090$ and $\theta=0.00098)$ compared with $G$. soja $(\pi=0.00114$ and $\theta=0.00164$ ) (Table 2), and secondly, landraces lacked $58 \%$ of the unique sequence variants present in G. soja despite that 2 novel SNPs happened (Fig. 1). However, the domestication bottleneck was slighter than that reported by Hyten et al. (2006). It maybe contributed to frequently communication among agricultural people since the Shang Dynasty or earlier and several domestication centers in China (Hymowitz and Newell 1981, Hymowitz 1970).

Intensive selection imposed in modern soybean breeding programs is generally thought to reduce 
genetic diversity of elite soybean cultivars (Gizlice and Burton 1994; Miranda et al. 2001; Concibido et al. 2004; Xiong et al. 2008), but Hyten et al. (2006) failed to find large effects of intensive selection based on DNA sequence diversity. In the present study, a clearly higher sequence diversity was found within cultivars $(\pi=0.00107$ and $\theta=0.00122)$ than in landraces ( $\pi=0.00090$ and $\theta=0.00098)$, especially for the sequence diversity in the coding region cultivars had sequence diversity increases of $\pi=0.00036$ and $\theta=0.00089$ compared to landraces (Table 2). In regard to unique SNPs, cultivars had two more than landraces in the overall sequence region, but seven more in the coding region (Fig. 1). This suggested that intensive selection has increased the sequence diversity for Rhg 4 in cultivars. The reason was probably the effect of selection in breeding programs on SCN disease, or other traits, such as oil content (Oil 1-1), protein content (Prot 17-4) and seed weight ( $S d w t 4-5)$ associated with yield, whose loci were close to Rhg4 (http://soybase.org/Marker DB/MapFeatureSearch.php?OutPutType=HTML\&map set=GmComposite2003\&MapName=A2\&FeatureType =All_Types\&FeatureStart=0\&FeatureStop=9999). Studies on association of Rhg4 alleles with SCN resistance, and on association mapping of SCN disease, yield and seed quality traits mentioned above on LG A2 (Chr 8) maybe helpful for explanation the effect of intensive selection on Rhg4 sequence diversity. In addition, incorporation of exotic germplasm from USA, France and Japan into breeding program (MOESM1) may also contribute to the high sequence diversity in cultivars.

Although there were pedigree relationships among some soybean elite varieties or lines in the population of cultivars (MOESM1), which may decrease the sequence diversity, DNA variations were detected among them (MOESM4), indicating possible different allele origins or recombination occurrence. For example, the four cultivars of Hefeng 25, Hefeng 23, Jilin 47 , and Suinong 14 were pedigree, but they were grouped into two clusters. Similar case also happened among the Kangxianchong 1, Kangxianchong 2 and Kangxianchong 3.

Acknowledgments This research was supported by National Natural Science Foundation of China (30490251 and 30471096), National Basic Research Program (2004CB117203), the State High-tech (863) (2006AA10A110, 2006AA10Z164, 2006AA 10A111), National Key Technologies R\&D Program in the 11th
Five-Year Plan (No.2006BAD13B05), and the Academy and Institute Foundation for Basic Scientific Research in Institute of Crop Science, Chinese Academy of Agricultural Sciences. We thank Dr Robert McIntosh for revision and suggestions on the manuscript.

Open Access This article is distributed under the terms of the Creative Commons Attribution License which permits any use, distribution, and reproduction in any medium, provided the original author(s) and the source are credited.

\section{References}

Anand SC, Shannon JG, Wrather JA, Arelli PR, Sleper DA, Young LD (2004) Registration of S97-1688 soybean germplasm line high in protein content and resistant to soybean cyst nematode. Crop Sci 44:698-699

Arelli AP, Wilcox JA (1997) Soybean germplasm resistant to races 1 and 2 of Heterodera glycines. Crop Sci 37:1367-1369

Arelli PR, Sleper DA, Yue P, Wilcox JA (2000) Soybean reaction to races 1 and 2 of Heterodera glycines. Crop Sci 40:824-826

Caldwell BE, Brim CA, Ross JP (1960) Inheritance of resistance of soybeans to the cyst nematode, Heterodera glycines. Agron J 52:635-636

Chang SJC, Doubler TW, Kilo VY, Abu-Thredeih J, Prabhu R, Freire V, Suttner R, Klein J, Schmidt ME, Gibson PT, Lightfoot DA (1997) Association of loci underlying field resistance to soybean sudden death syndrome (SDS) and cyst nematode (SCN) race 3. Crop Sci 37:965-971

Ching A, Caldwell KS, Jung M, Dolan M, Smith OS, Tingey S, Morgante M, Rafalski AJ (2002) SNP frequency, haplotype structure and linkage disequilibrium in elite maize inbred lines. BMC Genetics 3:e19

Clark RM, Schweikert G, Toomajian C, Ossowski S, Zeller G, Shinn P, Warthmann N, Hu TT, Fu G, Hinds DA (2007) Common sequence polymorphisms shaping genetic diversity in Arabidopsis thaliana. Science 317:338-342

Concibido VC, Denny RL, Boutin SR, Hautea R, Orf JH, Young ND (1994) DNA marker analysis of loci underlying resistance to soybean cyst nematode (Heterodera glycines Ichinohe). Crop Sci 34:240-246

Concibido VC, Lange DA, Denny RL, Orf JH, Young ND (1997) Genome mapping of soybean cyst nematode resistance genes in Peking, PI 90763, and PI 88788 using DNA markers. Crop Sci 37:258-264

Concibido VC, Diers BW, Arelli PR (2004) A decade of QTL mapping for cyst nematode resistance in soybean. Crop Sci 44:1121-1131

Coordinative Group of Evaluation of SCN (1993) Evaluation of soybean germplasm for resistance to race 1, 3 and 4 of the soybean cyst nematode. Soybean Sci 12(2):91-99

Diers BW, Cary TR, Thomas DJ, Nickell CD (2006) Registration of 'LD00-3309' soybean. Crop Sci 46:1384

Du ZQ, Tian ZY, Gao GJ, Zhou CJ, Wang MZ, Li ZX, Wu YK, Li XB (2006) Application and problems of using resistant 
varieties to $\mathrm{SCN}$ in Heilongjiang Province. Heilongjiang Agric Sci 3:32-34

Gizlice ZC, Burton TE (1994) Genetic base for North American public soybean cultivars released between 1947 and 1988. Crop Sci 34:1143-1151

Guo B, Sleper DA, Nguyen HT, Arelli PR, Shannon JG (2006) Quantitative trait loci underlying resistance to three soybean cyst nematode populations in soybean PI 404198A. Crop Sci 46:224-233

Gyuhwa C, Ram JS (2008) Broadening the genetic base of soybean: a multidisciplinary approach. Crit Rev Plant Sci 27:295-341

Hao XX, Jiang HL, Xu R, Wang JC (2003) Qihuang 28, a soybean variety with high oil content and resistance to soybean cyst nematode. Soybean Bull 5:26

Hauge BM, Wang ML, Parsons JD, Parnell LD (2001) Nucleic acid molecules and other molecules associated with soybean cyst nematode resistance. U.S. Pat Appl Publ No. 20030005491

Heer JA, Knap HT, Mahalingam R, Shipe ER, Arelli PR, Matthews BF (1998) Molecular markers for resistance to Heterodera glycines in advanced soybean germplasm. Mol Breed 4:359-367

Hymowitz T (1970) On the domestication of the soybean. Econ Bot 24:408-421

Hymowitz T, Newell CA (1981) Taxonomy of the genus Glycine, domestication and uses of soybeans. Econ Bot 35:272-288

Hyten DL, Song QJ, Zhu YL, Choi IY, Nelson RL, Costa JM (2006) Impacts of genetic bottlenecks on soybean genome diversity. Proc Natl Acad Sci USA 103:16666-16671

Hyten DL, Choi IY, Song Q, Shoemaker RC, Nelson RL, Costa JM, Specht JE, Cregan PB (2007) Highly variable patterns of linkage disequilibrium in multiple soybean populations. Genetics 175:1937-1944

Jang S, Van K, Kim MY, Gwag J, Jang H, Lee S (2004) SNP discovery and mapping of a major gene Rhg4 conferring resistance to soybean cyst nematode. Korean $\mathrm{J}$ Breed 36(2):76-80

Lai YC, Lin H, Fang WC, Yao ZC, Qi N, Wang QX, Yang XF, $\mathrm{Li} \mathrm{H}$ (2005) The excellent wild soybean resource screening, evaluation and utilization in Heilongjiang. China Agric Sci Bull 21:379-382

Lewers K, Heinz R, Beard H, Marek L, Matthews B (2002) A physical map of a gene-dense region in soybean linkage group A2 near the black seed coat and Rhg4 loci. Theor Appl Genet 104:254-260

Li YH, Zhang C, Gao ZS, Smulders MJM, Ma Z, Liu ZX, Nan HY, Chang RZ (2009) Development of SNP markers and haplotype analysis of the candidate gene for $r h g 1$, which confers resistance to soybean cyst nematode in soybean. Mol Breed 24:63-76

Li Y, Qi X, Chang R, Qiu L (2011) Evaluation and utilization of soybean fermplasm for resistance to cyst nematode in China. In: Sudaric A (ed) Soybean-molecular aspects of breeding. InTech, Croatia, pp 373-396

Lightfoot DA, Meksem K (2002) Isolated polynucleotides and polypeptides relating to loci underlying resistance to soybean cyst nematode and soybean sudden death syndrome and methods employing same. U.S. Pat Appl Publ No. 2002144310
Lindblad-Toh K, Winchester E, Daly MJ, Wang DG, Hirschhorn JN, Laviolette JP, Ardlie K, Reich DE, Robinson E, Sklar P (2000) Large-scale discovery and genotyping of single-nucleotide polymorphisms in the mouse. Nat Genet 24:381-386

Lu WG, Gai JY, Zheng YZ, Li WD (2006) Construction of a soybean genetic linkage map and mapping QTLs resistant to soybean cyst nematode (Heterodera glycines Ichinohe). Acta Agron Sin 32:1272-1279

Ma Y, Wang W, Wang L, Ma F, Wang P, Chang R, Qiu L (2006) Genetic diversity of soybean and the establishment of a core collection focused on resistance to soybean cyst nematode. J Integr Plant Biol 48:722-731

Mahalingam R, Skorupska HT (1995) DNA markers for resistance to Heterodera glycines I. Race 3 in soybean cultivar Peking. Breed Sci 45:435-443

Matson AL, Williams LF (1965) Evidence of a fourth gene for resistance to the soybean cyst nematode. Crop Sci 5:477

Matthews BF, Devine TE, Weisemann JM, Beard HS, Lewers KS, MacDonald MH, Park Y, Maiti R, Lin J, Kuo J, Pedroni MJ, Cregan PB, Saunders JA (2001) Incorporation of sequenced cDNA and genomic markers into the soybean genetic map. Crop Sci 41:516-521

Melito S, Heuberger AL, Cook D, Diers B, MacGuidwin AE, Bent AF (2010) A nematode demographics assay in transgenic roots reveals no significant impacts of the Rhg 1 locus LRR-kinase on soybean cyst nematode resistance. BMC Plant Biol 10:104. http://www.biomedcentral. com/1471-2229/10/104

Mengistu A, Kilen TC, Donald PA (2005) Registration of D981218 soybean germplasm line resistant to phytophthora rot (Rps2) and soybean cyst nematode races 3 (HG Type 0) and 14 (HG Type 1.3.6.7). Crop Sci 45:427-428

Miranda GV, Sediyama CS, Reis MS, Cruz CD (2001) Genetic diversity among elite Brazilian soybean cultivars with narrow genetic base. Crop Breed Appl Biotech 1:115123

Prabhu PR, Njiti VN, Bell-Johnson B, Johnson JE, Schmidt ME, Klein HJ, Lightfoot DA (1999) Selecting soybean cultivars for dual resistance to soybean cyst nematode and sudden death syndrome using two DNA markers. Crop Sci 39:982-987

Qiu LJ, Wang SM (2007) Characteristcs of Chinese soybean varieties released in 1993-2004. China Agriculture Press, Beijing

Rajesh PN, Muehlbauer FJ (2008) Discovery and detection of single nucleotide polymorphism (SNP) in coding and genomic sequences in chickpea (Cicer arietinum L.). Euphytica 162:291-300

Rao-Arelli AP, Anand SC, Wrather JA (1992) Soybean resistance to soybean cyst nematode race 3 is conditioned by an additional dominant gene. Crop Sci 32:862-864

Ravel C, Praud S, Canaguier A, Dufour P, Giancola S, Balfourier F, Chalhoub B, Brunel D, Linossier L, Dardevet M, Beckert M, Rousset M, Murigneux A, Charmet G (2007) DNA sequence polymorphisms and their application to bread wheat quality. Euphytica 158:331-336

Ruben E, Jamai A, Afzal J, Njiti VN, Triwitayakorn K, Iqbal MJ, Yaegashi S, Bashir R, Kazi S, Arelli P, Town CD, Ishihara H, Meksem K, Lightfoot DA (2006) Genomic analysis of the rhgl locus: candidate genes that underlie 
soybean resistance to the cyst nematode. Mol Genet Genomics 276:503-516

Schmid KJ, Sörensen TR, Stracke R, Törjék O, Altmann T, Mitchell-Olds T, Weisshaar B (2003) Large-scale identification and analysis of genome-wide single-nucleotide polymorphisms for mapping in Arabidopsis thaliana. Genome Res 13:1250-1257

Shannon JG, Lee J, Wrather JA, Sleper DA, Rouf Mian MA, Bond JP, Robbins RT (2009) Registration of S99-2281 soybean germplasm line with resistance to frogeye leaf spot and three nematode species. J Plant Regist 3:94-98

Tang H, Lewontin RC (1999) Locating regions of differential variability in DNA and protein sequences. Genetics 153:485-495

Tenaillon M, Sawkins MC, Long AD, Gaut RL, Doebley JF, Gaut BS (2001) Pattern of DNA sequence polymorphism along chromosome 1 of maize (Zea mays ssp. mays L.). Proc Natl Acad Sci USA 98:9161-9166

Todd JJ, Vodkin LO (1996) Duplications that suppress and deletions that restore expression from a chalcone synthase multigene family. Plant Cell 8:687-699

Van K, Hwang EY, Kim MY, Park HJ, Lee SH, Cregan PB (2005) Discovery of SNPs in soybean genotypes frequently used as the parents of mapping populations in the United States and Korea. J Hered 96:529-535

Vignal A, Milan D, SanCristobal M, Eggen A (2002) A review on SNP and other types of molecular markers and their use in animal genetics. Genet Sel Evol 34(3):275-305

Vuong TD, Sleper DA, Shannon JG, Nguyen HT (2010) Novel quantitative trait loci for broad-based resistance to soybean cyst nematode (Heterodera glycines Ichinohe) in soybean PI 567516C. Theor Appl Genet 121:1253-1266

Wang DG, Fan JB, Siao CJ, Berno A, Young P, Sapolsky R, Ghandour G, Perkins N, Winchester E, Spencer J (1998) Large-scale identification, mapping, and genotyping of single-nucleotide polymorphisms in the human genome. Science 280:1077-1082

Wang D, Arelli PR, Shoemaker RC, Diers BW (2001) Loci underlying resistance to race 3 of soybean cyst nematode in Glycine soja plant introduction 468916. Theor Appl Genet 103:561-566

Wang LX, Wang SY, Wang SR, Yuan M, Han DW, Wang F, Li XM (2007) Character and application of soybean variety Nenfeng No. 18 with high oil and resistant to cyst nematode. Heilongjiang Agric Sci 5:125-127

Webb DM, Baltazar BM, Rao-Arelli AP, Schupp J, Clayton K, Keim P, Deavis WD (1995) Genetic mapping of soybean cyst nematode race-3 resistance loci in the soybean PI437654. Theor Appl Genet 91:574-581
Weismann JM, Matthews BF, Devine TE (1992) Molecular markers located proximal to the soybean cyst nematode resistance gene, Rhg4. Theor Appl Genet 85:136-138

Winter SMJ, Shelp BJ, Anderson TR, Welacky TW, Rajcan I (2007) QTL associated with horizontal resistance to soybean cyst nematode in Glycine soja PI464925B. Theor Appl Genet 114:461-472

Wrather JA, Koenning SR (2009) Effects of diseases on soybean yields in the United States 1996 to 2007. Plant Health Progress. doi:10.1094/PHP-2009-0401-01-RS

Wrather JA, Anderson TR, Arsyad DM, Tan Y, Ploper LD, Porta-Puglia A, Ram HH, Yorinori JT (2001) Soybean disease loss estimates for the top ten soybean-producing countries in 1998. Can J Plant Pathol 23:115-121

Wu X, Blake S, Sleper DA, Shannon JG, Cregan P, Nguyen HT (2009) QTL, additive and epistatic effects for SCN resistance in PI 437654. Theor Appl Genet 118:1093-1105

Xiong DJ, Zhao TJ, Gai JY (2008) Parental analysis of soybean cultivars released in China. Scientia Agric Sin 41:2589-2598

Yamasaki M, Tenaillon MI, Bi IV, Schroederr SG, SanchezWilleda H, Doebley JF, Gaut BS, McMullen MD (2005) A large-scale screen for artificial selection in maize identifies candidate agronomic loci for domestication and crop improvement. Plant Cell 17:2859-2872

Young LD (1990) Soybean germplasm evaluated for resistance to races 3,5 , and 14 of soybean cyst nematode. Crop Sci 30:735-736

Yuan CP, Chang RZ, Qiu LQ (2006) Progress on genetic mapping and gene cloning of cyst nematode resistance in soybean. Chin Bull Bot 23:14-22

Yuan C, Zhou G, Li Y, Wang K, Wang Z, Li X, Chang R, Qiu L (2008) Cloning and sequence diversity analysis of GmHs $1^{\text {pro-1 }}$ in Chinese domesticated and wild soybeans. Mol Breed 22:593-602

Yue P, Sleper DA, Arelli PR (2001) Mapping resistance to multiple races of Heterodera glycines in soybean PI89772. Crop Sci 41:1589-1595

Zhang L, Dai Q (1992) Study on identification of soybean germplasm resistant to race 5 of soybean cyst nematode. Soybean Sci 11:79-82

Zhu YL, Song QJ, Hyten DL, Van Tassell CP, Matukalli LK, Grimm DR, Hyatt SM, Fickus EW, Young ND, Cregan PB (2003) Single-nucleotide polymorphisms in soybean. Genetics 163:1123-1134

Zhu Q, Zheng X, Luo J, Gaut BS, Ge S (2007) Multilocus analysis of nucleotide variation of Oryza sativa and its wild relatives: Severe bottleneck during domestication of rice. Mol Biol Evol 24:875-888 